\title{
A New Energy Efficient Cluster based Protocol for Wireless Sensor Networks
}

\author{
Mohamed Eshaftri, Ahmed Y.Al-Dubai, Imed Romdhani, *Muneer Beni Yassien \\ School of Computing, Edinburgh Napier University, 10 Colinton Road, Edinburgh EH10 5DT, UK \\ *Department of Computer Science, Jordan University of Science and Technology \\ Email: \{M.Eshaftri; A.Al-Dubai; I.Romdhani; M.BaniYassein\}@napier.ac.uk
}

\begin{abstract}
In Wireless Sensor Networks (WSNs), clustering techniques are usually used as a key effective solution to prolong the network lifetime by reducing energy consumption among the sensor nodes . Despite many works on clustering in WSNs this issue is still outstanding. However, the most existing solutions suffer from long and iterative clustering cycles. In an attempt to fil in this gap, we propose a new cluster-based protocol, referred to as Load-balancing Cluster Based Protocol (LCP) that introduces a new inter-cluster approach to increase network lifetime. This new protocol rotates continuously the election of the Cluster Head (CH) election in each cluster, and selects the node with the highest residual energy in each round. Extensive simulation experiments show that our proposed approach effectively balances the energy consumes among all sensor nodes and increases network lifetime compared to other clustering protocols.
\end{abstract}

Keywords-WSNs; Distributed clustering; lifetime; Routing; Network.

\section{INTRODUCTION}

$\mathbf{T}$ HE Wireless Sensor Network (WSN) technology has been one of the major avenues of networking and Internet of Things (IoT) due to their potential role in digitising smart physical environments [1]. WSNs composed of a large number of sensor nodes with limited battery power, which can be either densely or sparsely deployed in harsh and extreme environments, such as wild remote areas, natural habitats, and regions with access risk. On one hand, sensor nodes are usually battery-powered with limited operating time, and therefore they are highly sensitive to failure [2] [3]. On the other hand, the design an energy efficient WSNs protocol to prolonging the network lifetime is a challenging task due to the unique nature and strong networking constraints of wireless sensor networks [4].

The research community proposed different routing protocols to optimise the routing process in WSNs. Typically, the routing protocols for WSNs can be classified into three categories: flat, location and hierarchical based routing [5]. In flat routing, all nodes have identical functionality and they work together to sense and route [6]. Location based routing protocols rely on the position information of each node to discover and build optimal routing paths [7]. Compared to the two previous categories, in hierarchical routing approaches, the sensing field is subdivided into a set of administrative domains called clusters [8]. Each cluster has an organised leader or a root node called the ClusterHead $(\mathrm{CH})$. The primary aim of the $\mathrm{CH}$ is to collect data from attached and associated downstream nodes and forward it to the best next well-known hierarchical upper level upstream neighbour node. The data is forwarded in a hop-by-hop manner until it reaches the Base Station (BS). The BS can then send the data, using a wired or wireless Internet connection, to an end user located outside the sensing field [9][10].

A number of cluster protocols based on energy efficient have been proposed in the literature [11]. These approaches attempt to minimise energy consumption by reducing the transmission of redundant data. Clustering approaches focus primarily on the communication process during cluster organisation and $\mathrm{CH}$ election and neglect the effect of information processing on energy consumption. Hybrid Energy-Efficient Distributed (HEED) is one of the clustering protocols that uses both energy and communication costs to select $\mathrm{CHs}$ in a probabilistic manner. This protocol uses different inter-cluster approach in order to reduce energy consumption and to prolong the network lifetime [12].

In this paper, we present a new energy-aware distributed and dynamic clustering protocol, namely A Load-balancing Cluster Based Protocol (LCP). LCP addresses load balancing issues in cluster-based routing approaches. Given that cluster-based protocols require regular re-clustering for balancing energy consumption. However, re-clustering process in interval time for entire network increases the network overhead and consequently decreases the network operation time. The proposed model provides a pre-defined interval of time at the beginning of every round to select the $\mathrm{CH}$. This delays the frequency of the re-clustering messages received from the BS. If the sensor nodes do not receive the $\mathrm{BS}$ message, the $\mathrm{CHs}$ continues rotating the leadership among them within the same members of cluster by electing the node with the highest residual energy each round. The performance evaluation of LCP is examined in depth and compared to HEED [12], LEACH [13] and RHEED [14]. Obtained results demonstrate that LCP enhances the network lifetime by $15 \%$.

The rest of the paper is organised as follows. In Section II, we review a set of up-to-date clustering algorithms proposed for WSNs. Section III presents the features of the new LCP protocol. Section IV presents a detailed description of the simulation environment and the simulation results. Finally, section $\mathrm{V}$ reviews the entire study and offers conclusions and recommendations for future work. 


\section{RELATED WORK}

Different cluster-based approaches have been proposed by the research community to address the challenging issues of WSNs. Some of these approaches are as follows:

\section{A. Low Energy Adaptive Clustering Hierarchy (LEACH)}

Heinzelman, et al. proposed the first well-known clustering LEACH protocol [13]. This protocol was targeted at prolonging the lifetime of WSNs and reducing the energy consumption of sensor nodes. From an algorithmic point of view, LEACH is hierarchical, probabilistic, distributed and single-hop protocol. It forms clusters based on the strength of received signal, while $\mathrm{CH}$ nodes act as default gateways to the BS, as illustrated in Fig. 1. In LEACH protocol, nodes make autonomous decisions without relying on a centralised third party entity. In addition, all nodes have an equal opportunity to become CHs. Initially, a node generating a random number between (0-1) to be a $\mathrm{CH}$ by comparing it with a threshold value $\mathrm{T}(\mathrm{n})$, calculated using Equation (1).

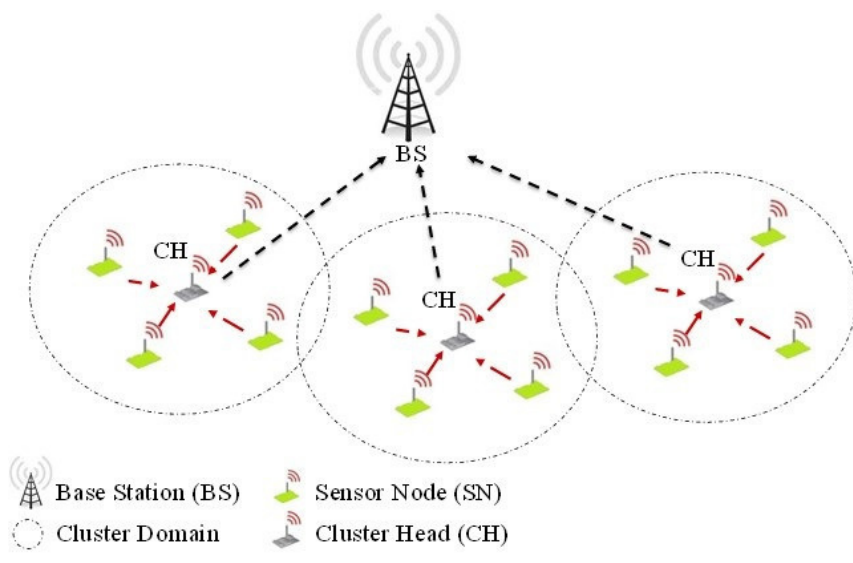

Fig. 1. Basic LEACH topology [11]

Nodes with a random number lower than $\mathrm{T}(\mathrm{n})$ then become $\mathrm{CHs}$. Each elected $\mathrm{CH}$ broadcasts an advertisement to non$\mathrm{CHs}$ to form a cluster.A non- $\mathrm{CH}$ node selects a $\mathrm{CH}$ that expending the least energy for communication.

$$
T(n)=\left\{\begin{array}{c}
\frac{p}{1-p\left(\operatorname{rmod} \frac{1}{p}\right)} \\
0
\end{array} \quad \text { if } n \in G\right.
$$

Where $\mathrm{p}$ is the desired percentage of nodes to be $\mathrm{CH}$; $r$ is the current round; $G$ is the set of nodes that have not been cluster heads during the last $1 / \mathrm{P}$ rounds.

Generally, LEACH provides a good model for energy consumption while providing an equal probability for node to be elected $\mathrm{CHs}$. Once chosen as a $\mathrm{CH}$, a sensor node cannot be reselected in a subsequent round. Moreover, LEACH avoids unnecessary collisions between $\mathrm{CHs}$ because it uses the Time Division Multiple Access (TDMA) protocol. Despite its generally good performance, LEACH also has some clear limitations. It uses single-hop communication which limits its scalability. In addition, the probabilistic election mechanism of CHs may lead to either high concentrations of $\mathrm{CHs}$ in one part of the network, or to orphan nodes (nodes without $\mathrm{CHs}$ in their neighbourhood.

\section{B. LEACH-Centralised (LEACH-C)}

To address the shortcomings of LEACH with respect to determining each $\mathrm{CHs}$ location and number rounds, a new version of LEACH, named LEACH Centralised (LEACH-C) proposed [15]. In the new version, the BS decides which sensor nodes are eligible to become $\mathrm{CHs}$ and form a cluster. Each node transmits its location and energy level to the BS, which in return calculates the average energy level for the network and eliminates the nodes with remaining energy levels below this average, to form the set of $\mathrm{CHs}$ for that round. In the centralised algorithm the energy load is distributed among all nodes equally, where the numbers of $\mathrm{CHs}$ are specified and the network is divided into optimum and equal sized clusters. However, the construction of clusters with an equal number of nodes in each cluster is not guaranteed in this protocol, and it is not always possible for nodes distant from the BS to send information about their status.

\section{CHybrid Energy-Efficient Distributed (HEED)}

O. Younis et al. [12] Introduced HEED clustering protocol. In this protocol, the authors enhanced LEACH protocol by introducing two basic parameters to elect the $\mathrm{CHs}$. The first main parameter concerns the remaining energy of each node, and the second parameter is the intra-cluster "communication cost". For example, the cost can be a function of neighbour proximity or cluster density, that can calculated using Equation (2). Unlike LEACH, HEED protocol the $\mathrm{CH}$ nodes are not randomly selected. Only nodes with high levels of remaining energy can become $\mathrm{CH}$ nodes. In addition, when two nodes are within each other's cluster range, the probability of both becoming cluster heads is negligible. In comparison to LEACH, in HEED, the CHs are well distributed throughout the network. However, this protocol cannot fix the cluster count in each round. In addition, the energy consumption is not balanced, because more $\mathrm{CHs}$ could be generated more than expected, which creates massive overheads due to multiple election rounds.

$$
C H_{\text {prob }}=C_{\text {prob }} \times \frac{E_{\text {residual }}}{E_{\max }}
$$

Where: $C_{\text {prob }}$ is an initial percentage of cluster heads among all $\mathrm{n}$ nodes, $E_{\text {residual }}$ is the estimated current energy of the node, $E_{\max }$ is the referenced maximum energy (corresponding to a fully charged battery).

\section{Distributed Energy-Efficient Clustering (DEEC)}

Li Qing et al. [16] proposed the DEEC algorithm for WSN to improve HEED performances. In DEEC, the CHs are selected with a probability based on the residual energy of each node and the average energy of the network. The authors of this algorithm assumed that nodes would have different amounts of energy. With the adaptive values, the sensor 
nodes determine their role probabilistically in each round. The main drawback of DEEC is that each node demands global knowledge from the network, which increases the overheads.

\section{E. Rotated Hybrid, Energy-Efficient and Distributed (R- HEED)}

W. Mardini et al. [14] introduced R-HEED. With this protocol, the authors improved the performance of HEED by applying a different inter-cluster approach. The new approach conducts the cluster reformation based on certain rules. At the start of setup phase on every round, the $\mathrm{CHs}$ node must delay for period of time waiting for a cluster reformation message from the sink .if the cluster reformation message not received, each cluster persevere with rotating the cluster head task in the same cluster. However, randomly rotating the $\mathrm{CH}$ does not take into account energy consumption.

\section{F. Distributed weight based energy efficient hierarchical clus- tering protocol (DWEHC)}

P Ding et al. [18] proposed a new protocol called DWEHC that improves HEED performances. Their primary aim was to to improve energy consumption by forming balanced cluster sizes and improving intra-cluster routing. Each sensor node begins broadcasting its $(\mathrm{x}, \mathrm{y})$ coordinates to search for its neighbour. After finding neighbouring nodes in its area, each node calculates its weight. Weight is the only parameter calculated locally and used for $\mathrm{CH}$ election; it is represented by weight in DWEHC as defined by Equation 3.The node with the largest weight is selected as a $\mathrm{CH}$. The ordinary nodes become child nodes by joining $\mathrm{CH}$. The nodes at this stage, are considered first level members because they have a direct link to the $\mathrm{CH}$. As the child nodes are further divided into levels (level 1, level 2, etc.) the total number of levels is seen to depend on the cluster range and the minimum $\mathrm{CH}$ energy. Like HEED, DWEHC is a fully distributed clustering protocol with a more balanced $\mathrm{CH}$ distribution. In addition, its clustering process does not rely on network size. However, this protocol cannot increase its energy efficiency give its intercluster communication function and the large control message overheads.

$$
W_{\text {weight }(s)}=\left(\sum_{u \in N \alpha, c(s)}\right) \frac{(R-d)}{6 R} \times \frac{\operatorname{Eresidual}(s)}{\operatorname{Einitial}(s)}
$$

Where: $\mathrm{R}$ is the cluster range, $\mathrm{d}$ is the distance from node $\mathrm{s}$ to neighbouring node; $E_{\text {residual (s) }}$ is the residual energy in the nodes; $E_{\text {initial }(s)}$ is the initial energy in the nodes.

\section{G. Power-Efficient and Adaptive Clustering Hierarchy (PEACH)}

The majority of existing clustering protocols consume large amounts of energy, incurred by cluster formation overheads and fixed-level clustering. This is especially true when sensor nodes are densely deployed. To address this problem, Sangho Yi et al. [17] proposed PEACH protocol to reduce energy consumption, that improve the network lifetime.In PEACH protocol, a node selected as $\mathrm{CH}$ when the packet received was for the that node. When the packet is received by a different node and is not the destination for the packet, the node will join the destination of that packet. Simulation results showed that PEACH consumes lower energy and prolonges the network lifetime comparative to the LEACH, and HEED protocols. However, the network is not very scalable, because all the nodes must have global knowledge of the network.

\section{THE LCP CLUSTERING PROTOCOL}

The proposed scheme builds on the success of the HEED protocol. The clustering phase of the HEED protocol has been modified to make it more energy-efficient. The modified version is named A Load-balancing Cluster Based Protocol (LCP). In LCP, the clustering operation is divided into several rounds, each round has two phases: the setup and the steadystate phase. LCP is similar to HEED in terms of the following features:

- The elected CHs sent advertisement message within cluster range.

- The cluster formation "setup phase" finish in $\mathrm{O}(1)$ iterations.

- Each node become member only to one cluster and communicates directly with its $\mathrm{CH}$.

- Through the cluster formation process, Nodes can become either a tentative_CH or a final_CH, or it can be covered.

- At the end of the clustering procedure, $\mathrm{CHs}$ node forms a network backbone. Thus, the data is forwarded in hopby-hop through $\mathrm{CHs}$ until it reaches the BS.

- The steady state phase for LCP protocol is alike HEED, and $\mathrm{CH}$ election is done as part of an iterative process.

The setup phase is divided into four phases: 1) Initialise phase, 2) Repeat phase, 3) Finalise phase and, 4) Rotation phase. The following steps describe the proposed phases, which are illustrated in Fig. 2.

1) Initialise phase: At the beginning of this phase, nodes exchange their information with neighbours in order to computes its cost. Unlike HEED, the costs are exchanged through the cluster head message. The LCP algorithm sets an initial percentage of node become Cluster head $C_{\text {prob. }}$. Thus, each sensor node establishes its probability of becoming a $\mathrm{CH}$ based on the reaming energy $\mathrm{CH}_{\text {prob }}$ according to HEED.

2) Repeat phase "Main Processing": Each node in this phase is subject to a delay time, in which it can decide whether the node will be elected as candidate $\mathrm{CH}$ node "tentative_CH". If the node not elected as tentative_ $\mathrm{CH}$, it will declare itself a cluster head node "final_CH". The final_CH node broadcast a cluster_head_msg (Node ID, tentative, cost) within cluster range.

3) Finalize phase: During this phase, most sensor nodes declare itself either a cluster head node or a member node. If node received a cluster head advertise message, it will join the final_CH with the lowest cost. The node neither final_CH or has not received cluster head advertise message, it will declare itself a final_CH node. 

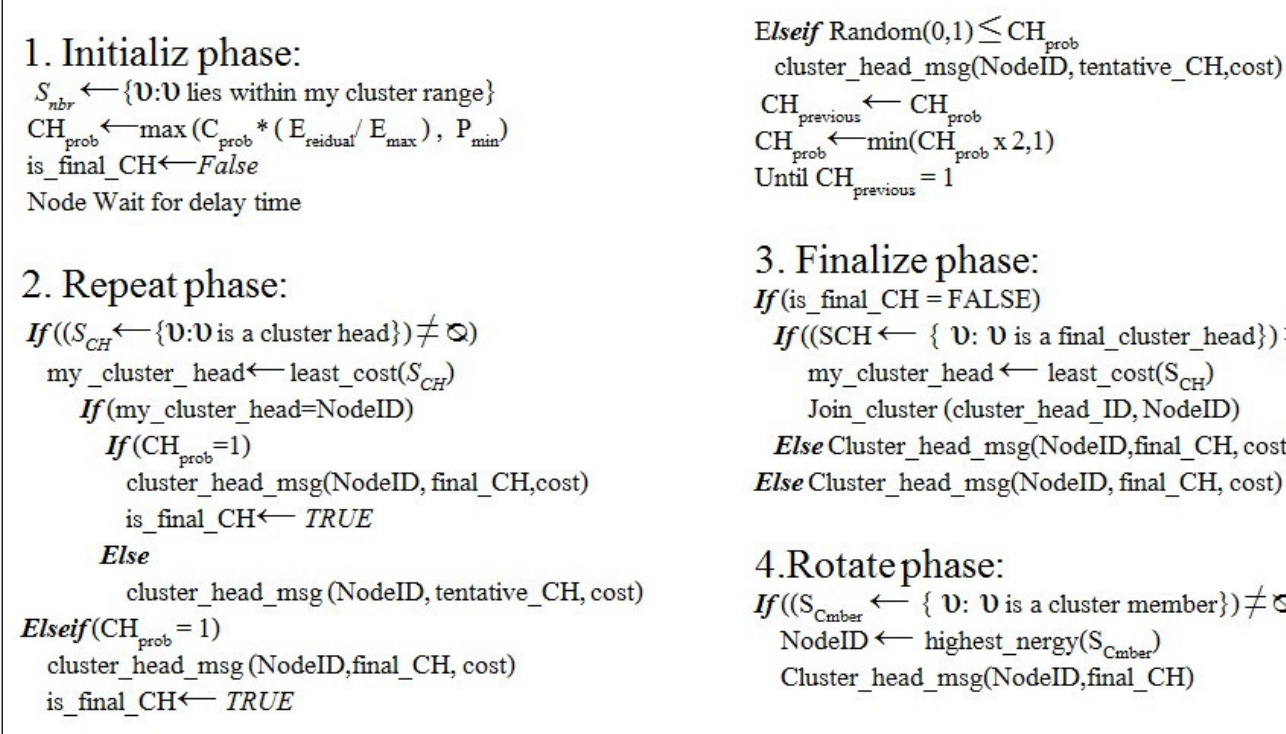

3. Finalize phase:

If (is_final_CH $=$ FALSE)

If $\left(\left(\mathrm{SCH}^{-} \leftarrow\{\mathrm{V}: \mathrm{v}\right.\right.$ is a final_cluster_head $\left.\left.\}\right) \neq Q\right)$

my_cluster_head $\longleftarrow$ least_cost $\left(\mathrm{S}_{\mathrm{CH}}\right)$

Join_cluster (cluster_head_ID, NodeID)

Else Cluster_head_msg(NodeID,final_CH, cost)

Else Cluster_head_msg(NodeID, final_CH, cost)
4.Rotate phase:
If $\left(\left(\mathrm{S}_{\text {Cmber }} \longleftarrow\{\mathrm{v}: \mathrm{v}\right.\right.$ is a cluster member $\left.\left.\}\right) \neq Q\right)$
NodeID $\longleftarrow$ highest_nergy $\left(\mathrm{S}_{\mathrm{Cmber}_{2}}\right)$
Cluster_head_msg(NodeID,final_CH)

Fig. 2. A Load-balancing Cluster Based algorithm.

4) Rotate phase: After elect the $\mathrm{CH}$ sode and form clusters in the first round, each $\mathrm{CH}$ constructs a turning schedule for its member when it becomes a $\mathrm{CH}$. The turns are sorted based on residual energy in the sensor node. Node with the highest residual energy will be the first candidate to become a $\mathrm{CH}$ for next round. Therefore, at begging of the next round unlike HEED protocol is not necessary to re-cluster the network. Node within the same cluster in subsequent rounds continues rotating the $\mathrm{CH}$ role between them, by selecting the node with the highest residual energy every round. When first cluster finishes the rotating process, it inform the BS by sending re-form cluster message via multi-hop route. BS re-broadcast the message among the nodes inform them to start a new cluster process, See Rotate phase in Fig. 3.

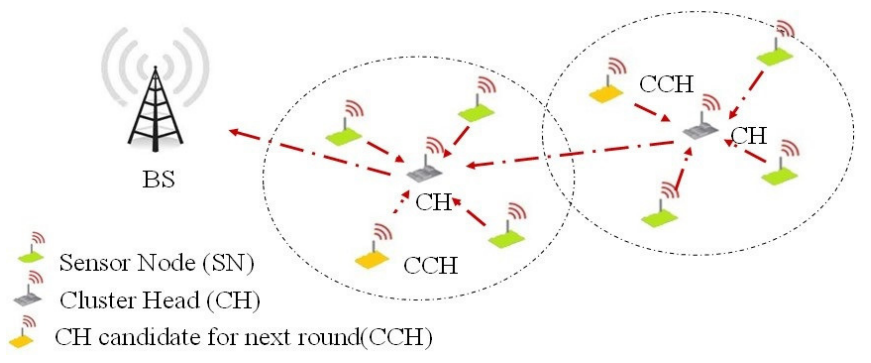

Fig. 3. A Load-balancing Cluster Based Protocol Rotate phase.

\section{PERFORMANCE EVALUATION}

In this section, we evaluate the performance of the LCP mechanism by using open source Castalia simulator [19]. We consider a sensor network, composed of (100-350) sensor nodes, which are randomly deployed in a playground of $200 \mathrm{mX} 200 \mathrm{~m}$ square region. All sensor nodes are fixed and homogeneous and with limited stored energy. Nodes are not equipped with GPS-capable antennae. The BS is placed at the center of the sensor field. The energy consumption for each sensor node is calculated by data transmission and aggregation per round. The energy efficiency of LCP is compared against LEACH, HEED, R-HEED. Simulation parameters are given in Table I.

TABLE I

PARAMETER SETTINGS

\begin{tabular}{|c|c|}
\hline Parameter & Value \\
\hline Deployment field & 200 X 200 m \\
\hline Data packet size & 200 bytes \\
\hline Control packet & 25 bytes \\
\hline Number of node & $100-350$ \\
\hline Initial cluster radius $(\mathrm{RC})$ & $25 \mathrm{~m}$ \\
\hline Sink position & $(0,0)$ \\
\hline Initial energy & $25 \mathrm{~J}$ \\
\hline Threshold distance $\left(d_{0}\right)$ & $75 \mathrm{~m}$ \\
\hline Deployment method & Uniform, Random \\
\hline Rotated time $\left(T_{r}\right)$ & 20 Sec \\
\hline Radio model & CC4220 \\
\hline
\end{tabular}

We use in this paper the residual energy matric,and the network lifetime metric to evaluate the performance of our protocol. The residual energy metric is computed by the average energy remaining in all nodes at a specific round. The network lifetime metric is based on WSN applications require.For example, applications require that all node must work to ensure the network has good coverage. Thus, the network lifetime metric for these applications should be measured according to the lifetime of the shortest-living node. Some Other applications they only need a specific percentage of nodes have 
to remain alive to achieve the applications requirement [17]. Therefore, the network lifetime in our protocol ,is measured by following three different metrics.

1) First Node Die (FND): is defined as time elapsed in rounds until the first node has consumed all available energy.

2) Half Nodes Die (HND): is defined as time elapsed in rounds until half of the nodes have consumed all available energy stores.

3) Last Node Dies (LND): is defined as time elapsed in rounds until all the nodes have exhausted their entire energy supply.

The "round" definition in our paper refer to the time interval in seconds befor the network statr a new cluster process. Therfore , no difference between the round concepts in LCP , and HEED in terms of time.In LCP protcol we specified a round time of 20 seconds.

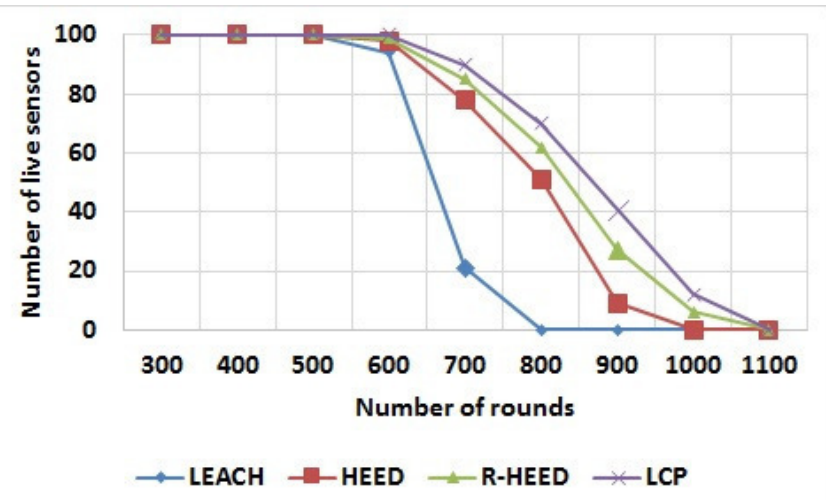

Fig. 4. Number of alive sensors Vs numbers of rounds for LEACH, HEED, R-HEED and LCP.

Fig. 4 . demonstrates the total number of nodes remaining alive following the simulation round. LCP increases the network lifetime compared to its peers. Fig. 5. demonstrates the relationship between the remaining energy and the number of nodes. It is evident that LCP consumes the least amount of energy. Furthermore, how the increasing number of the nodes affects the lifetime of each protocol has been evaluated. . Fig.6 demonstrates the network lifetime until the first node dies, when the number of nodes varies between (150-350).

Figure.7 and 8 also reveals the same comparisons to define the network lifetime.In Fig.7 shows the number of rounds until half of the nodes die, while Fig.8 show the number of rounds until the last node dies. It is evident that the network lifetime improves when the number of nodes increases in all protocols. Figs 6, 7 and 8 show that in all three cases LCP protocol performs better than the rest of the protocols. This advancement is caused by the rotating process of the cluster heads within the same cluster.

Consequently, the rotating process leads to reduc the energy consumption among the nodes, and increasing the network lifetime. It can be easily observed from the simulation results that when the number of the nodes increases the percentage

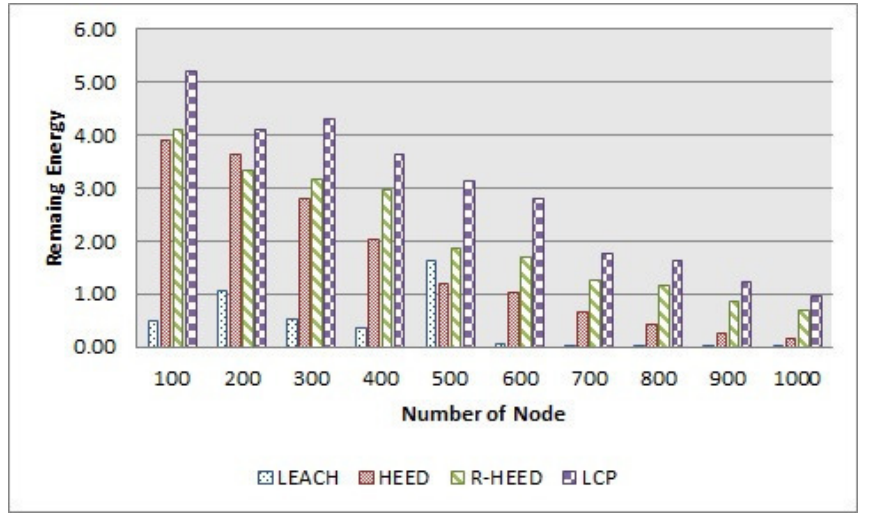

Fig. 5. Total remaining energy in LCP in comparison with HEED, R-HEED.

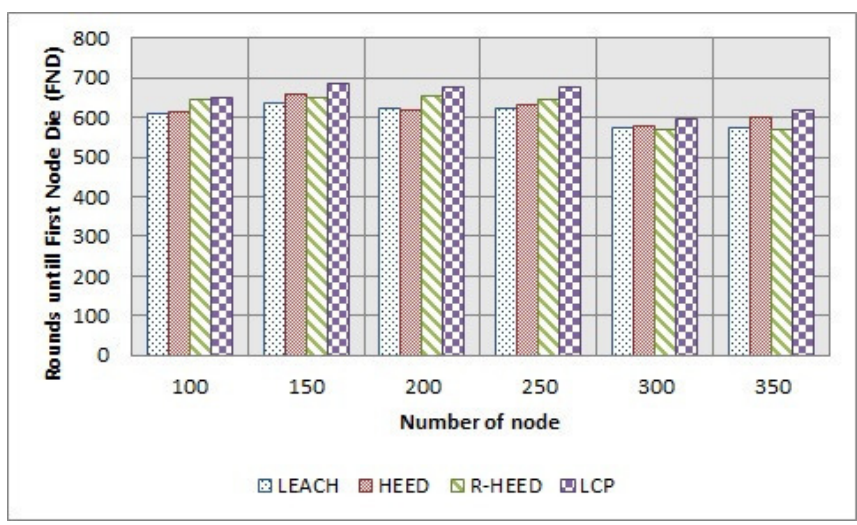

Fig. 6. Comparing LEACH, HEED, R-HEED and LCP using different number of node for FND metric.

improvement also increases. Therefore, it can be reasoned that when Increase the amount of nodes it reduce the energy consumed during the setup phase.Thus, the energy saved as a result of this new clustering scheme will be maximised, which will improve the networkâĂŹs lifetime.

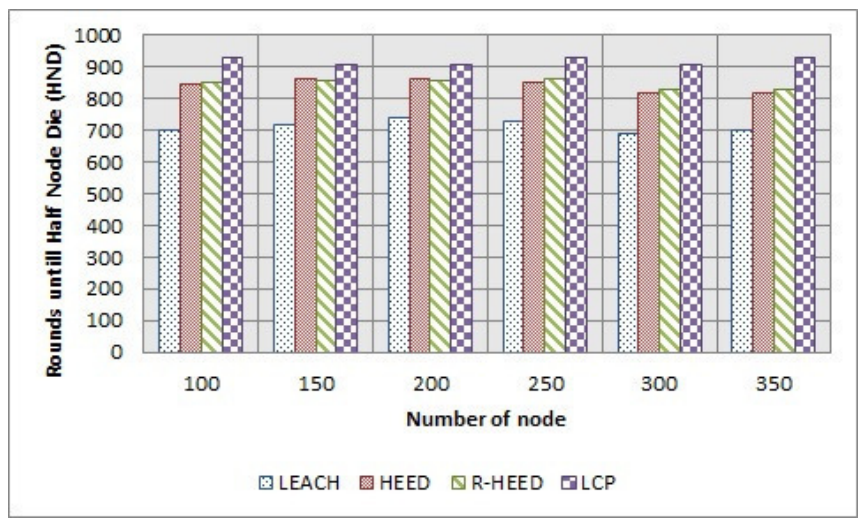

Fig. 7. Comparing LEACH, HEED, R-HEED and LCP using different number of node for HND metric. 


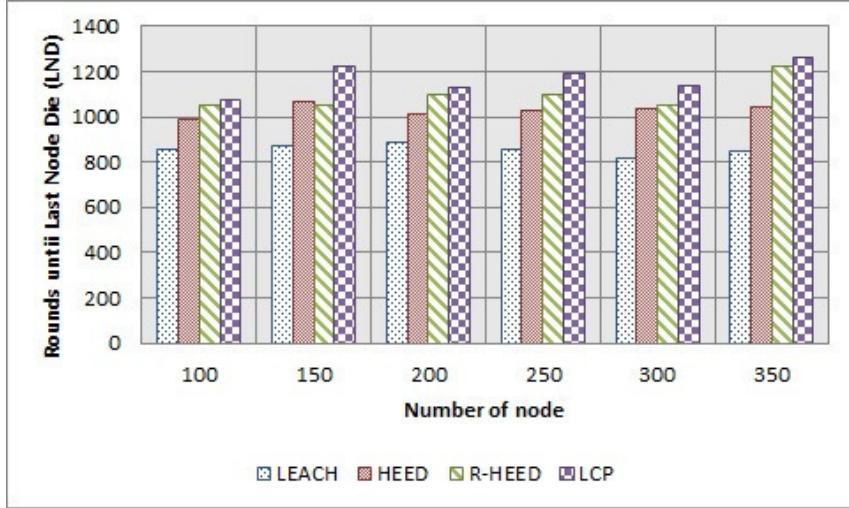

Fig. 8. Comparing LEACH, HEED, R-HEED and LCP using different number of node for LND metric.

\section{CONCLUSIONS AND FUTURE WORK}

In this paper, the clustering scheme Load-balancing Cluster Based Protocol (LCP) for wireless sensor networks was proposed as a more energy-efficient protocol. The main contribution of the LCP protocol is its ability to continue rotating the cluster head $(\mathrm{CH})$ role between nodes within the same cluster, by selecting the node with the highest residual energy to become a $\mathrm{CH}$ for the next round. We compared and evaluated the LCP protocol performance with well-known Energy Efficient clustering protocols, which is have the same aim increase the network lifetime. The simulation results showed that LCP protocol significant balance the energy consumption among the entire node and achieves an obvious improvement to the network's lifetime by $15 \%$.

Finally, we evaluated our protocol performance in term of energy consumption. Hence our future work we plane to investigate the performance of LCP according to other networking metrics, such as packet delivery ratio and endto-end delay.

\section{REFERENCES}

11 C. Perera, A. Zaslavsky, P. Christen, and D. Georgakopoulos,"Context aware computing for the internet of things: A survey," IEEE Commun. Surv. Tutorials, vol. 16, no. 1, pp. 414-454, 2014 http://dx.doi.org/10.1109/SURV.2013.042313.00197

[2] Z. Manap, B. M. Ali, C. K. Ng, N. K. Noordin, and A. Sali," A review on hierarchical routing protocols for wireless sensor networks," Wireless Personal Communications, vol. 72, no. 2, pp. 1077-1104, 2013 http://dx.doi.org/10.1007/s11277-013-1056-5

[3] R. N. Enam , M. Imam and R. I. Qureshi "Energy Consumption in Random Cluster Head selection Phase of WSN" 2012 IACSIT Hong Kong Conferences IPCSIT vol. 30 (2012) IACSIT Press, Singapore.
[4] M. Shokouhifar and A. Jalali, "A new evolutionary based application specific routing protocol for clustered wireless sensor networks," AEU - Int. J. Electron. Commun., vol. 69, no. 1, pp. 432-441, 2015 http://dx.doi.org/10.1016/j.aeue.2014.10.023

[5] A. Rahman, S. Anwar, I. Pramanik, and F. Rahman, "A Survey on Energy Efficient Routing Techniques in Wireless Sensor Network," 15th International Conference on Advanced Communications Technology (ICACT 2013). Pyeong Chang, South Korea, pp. 200-205, January 2013.

[6] C. Intanagonwiwat, R. Govindan, D. Estrin, J. Heidemann, and F. Silva, "Directed diffusion for wireless sensor networking," Networking, IEEE/ACM Trans., vol. 11, no. 1, pp. 2-16, 2003. http://dx.doi.org/10.1109/TNET.2002.808417

[7] $\mathrm{Y}$ Xu, J. Heidemann, and D. Estrin, "Geography-Informed Energy Conservation for Ad Hoc Routing," Proc. ACM/IEEE Intl Conf. Mobile Computing and Networking (MOBICOM), pp. 70-84, July 2001 http://dx.doi.org/10.1145/381677.381685

[8] S. Gupta, N. Jain, and P. Sinha, "Clustering Protocols in Wireless Sensor Networks: A Survey,” in International Journal of Applied Information Systems (IJAIS) âĂS ISSN : 2249-0868 Foundation of Computer Science FCS, New York, USA vol. 5, no.2, pp.41-50, January 2013.

[9] S. P. Singh and S. C. Sharma, "Cluster Based Routing Algorithms for Wireless Sensor Networks," International Journal of Engineering \& Technology Innovations (IJETI), vol. 1, no 4, November 2014.

[10] A. Marya, A. Kumar, and C. Mohan, "Energy efficient hetero geneous leach with enhanced stability for wireless sensor network systems," International Journal of Applied Science and Engineering Research (IJASER), vol. 3, no. 5, pp. 920-931, 2014 http://dx.doi.org/10.6088/ijaser.030500002

[11] D. J. Dechene, A. El Jardali, M. Luccini, A. Sauer, "A Survey of Clustering Algorithms for wireless Sensor Networks," Information and Automation for Sustainability (ICIAFS), 4th International Conference Publication Year: 2008 , pp. 295-300.

[12] O. Younis and S. Fahmy, "HEED: A Hybrid, Energy-Efficient, Distributed Clustering Approach for Ad Hoc Sensor Networks," IEEE Trans. Mobile Computing, vol. 3, no. 4, pp. 366-379, Oct.-Dec. 2004. http://dx.doi.org/10.1109/TMC.2004.41

[13] W. Heinzelman, A. Chandrakasan and H. Balakrishnan, "EnergyEfficient Communication Protocol for Wireless Microsensor Networks," Proc. 33rd Hawaii Intl. Conf. Sys. Sci., Jan. 2000. http://dx.doi.org/10.1109/HICSS.2000.926982

[14] W. Mardini, M. B. Yassein, Y. Khamayseh, and B. a. Ghaleb, "Rotated hybrid, energy-efficient and distributed (R-HEED) clustering protocol in WSN,” WSEAS Trans. Comm , vol. 13, pp. 275-290, 2014.

[15] W. Heinzelman, A Chandrakasan, and H. Balakrishnan, "An Application-Specific Protocol Architecture for Wireless Microsensor Networks," IEEE Trans. Wireless Comm., vol. 1, no. 4, pp. 660- 670, Oct. 2002. http://dx.doi.org/10.1109/TWC.2002.804190

[16] L. Qing, Q. Zhu, and M. Wang, "Design of a distributed energy-efficient clustering algorithm for heterogeneous wireless sensor networks," Computer Commun., vol. 29, pp. 2230-2237, 2006. http://dx.doi.org/10.1016/j.comcom.2006.02.017

[17] S. Yi, J. Heo, Y. Cho, and J. Hong, "PEACH: Power-efficient and adaptive clustering hierarchy protocol for wireless sensor networks," Comput. Commun., vol. 30, no. 14-15, pp. 2842-2852, Oct. 2007. http://dx.doi.org/10.1016/j.comcom.2007.05.034

[18] P. Ding, J. Holliday, and A. Celik, "Distributed energy-efficient hierarchical clustering for wireless sensor networks," in Proceedings of the IEEE International Conference on Distributed Computing in Sensor Systems (DCOSS âÁŹ05), vol. 3560, pp. 322-339, June 2005. http://dx.doi.org/10.1007/11502593_25

[19] A.Boulis, "A Simulater for Wireless Sensor Networks and Body Area Network," (Castalia), March 2011, https://castalia.forge.nicta.com.au, (Accessed: 9 February 2014) 